Preface

\title{
Hot Topics VI
}

\author{
Emmanuel J. Favaloro, PhD, FFSc (RCPA) ${ }^{1}$ \\ ${ }^{1}$ Department of Haematology, Institute of Clinical Pathology and \\ Medical Research (ICPMR), Westmead Hospital, Westmead, Australia
}

Semin Thromb Hemost 2014;40:713-717.

Welcome to the sixth issue compilation of Seminars in Thrombosis and Hemostasis entitled "Hot Topics."1-5 The previous issues have been very popular with our readership, as identified in subsequent analyses. ${ }^{6-11}$ Although Seminars in Thrombosis \& Hemostasis is primarily a theme-driven publication, the occasional opportunity arises to publish composite issues containing more wide-ranging articles of current interest and controversy. This opportunity presents itself once again as a result of several events, including the most recent (2014) Eberhard F. Mammen Young Investigator Awards. ${ }^{12}$ Accordingly, I am very pleased to present the latest "Hot Topics" issue (Part VI), including contributions from three of six of our 2014 Eberhard F. Mammen Young Investigator Award winners. ${ }^{12}$ The other award winners will provide contributions that will be published in a subsequent issue of this journal. The remaining articles from this Hot Topics issue derive from various other contributors, and we have a very interesting potpourri of material to share with our readership.

The issue begins with a seminal contribution from the International Society on Thrombosis and Hemostasis Steering Committee for World Thrombosis Day, and on thrombosis as a major contributor to global disease burden. ${ }^{13}$ This contribution is being published jointly within several leading hemostasis/thrombosis journals to celebrate the inaugural "World Thrombosis Day" on October 13, 2014, and indeed, we have delayed publication of this issue of the journal specifically to coincide with this date. Seminars in Thrombosis \& Hemostasis is proud to be a part of this important initiative, and this article is also being published online as an open access paper, entirely free for all to download. The writing group of Raskob et al have performed a systematic review of the literature on the global disease burden due to venous thromboembolism (VTE) in low-, middle-, and high-income countries. Studies from Western Europe, North America, Australia, and Southern Latin America (Argentina) yielded consistent results with annual incidences ranging from 0.75 to 2.69 per 1,000 individuals in the population. The incidence increased to between

Address for

correspondence Emmanuel J. Favaloro, PhD, FFSc (RCPA),

Department of Haematology,

Institute of Clinical Pathology and

Medical Research (ICPMR),

Westmead Hospital, Westmead, Australia (e-mail: emmanuel.

favaloro@swahs.health.nsw.gov.au).
2 and 7 per 1,000 among those aged 70 years or more. Of special note, and of special relevance to our readership, is the fact that the increasing burden of thrombosis with aging was recently highlighted in this journal. ${ }^{14}$ Indeed, one of the guest editors to this previous issue of the journal ${ }^{14}$ was also a contributor to the current thrombosis burden review. ${ }^{13}$ The data presented in this review, as associated with age-related VTE, are summarized in - Fig. 1. Although the incidence of VTE is lower in individuals of Chinese and Korean ethnicity, their total disease burden is not low because of their population aging. ${ }^{13}$ VTE associated with hospitalization was, perhaps unsurprisingly, the leading cause of disability-adjusted life years (DALYs) lost in low- and middle-income countries, and the second leading cause in high-income countries. VTE associated with hospitalization was responsible for more DALYs lost than nosocomial pneumonia, catheter-related bloodstream infections, and adverse drug events. The authors conclude that VTE causes a major burden of disease in all countries, denoting low-, middle-, and high-income countries. In summary, thrombosis is a common pathology underlying ischemic heart disease, ischemic stroke, and VTE. Providing detailed data on the global burden of VTE should enable informed policy and resource allocation in health systems, and to assess if improved utilization of preventive measures will reduce the burden.

This issue of the journal continues the topic of thrombosis and related disease burden with an Australian-based contribution on the topic of VTE in tropical Australia and in Indigenous Australians. ${ }^{15}$ There is a paucity of data on the incidence VTE in this population of Australians, and accordingly, the authors have conducted a retrospective review of all cases of deep vein thrombosis and pulmonary embolism over a 24-month period in two major hospitals of the Northern Territory. A total of 429 VTE diagnoses were recorded over this period and 71 of 429 (17\%) patients were Indigenous Australians. The overall incidence rate was 0.9 per 1,000 person-year for the population of the Northern Territory with a rate of 0.5 per 1,000 person-year for Indigenous
Copyright $\odot 2014$ by Thieme Medical Publishers, Inc., 333 Seventh Avenue, New York, NY 10001, USA. Tel: +1(212) 584-4662.
DOI http://dx.doi.org/ 10.1055/s-0034-1394110. ISSN 0094-6176.
Editor, Emmanuel J. Favaloro, PhD, FFSC (RCPA). 


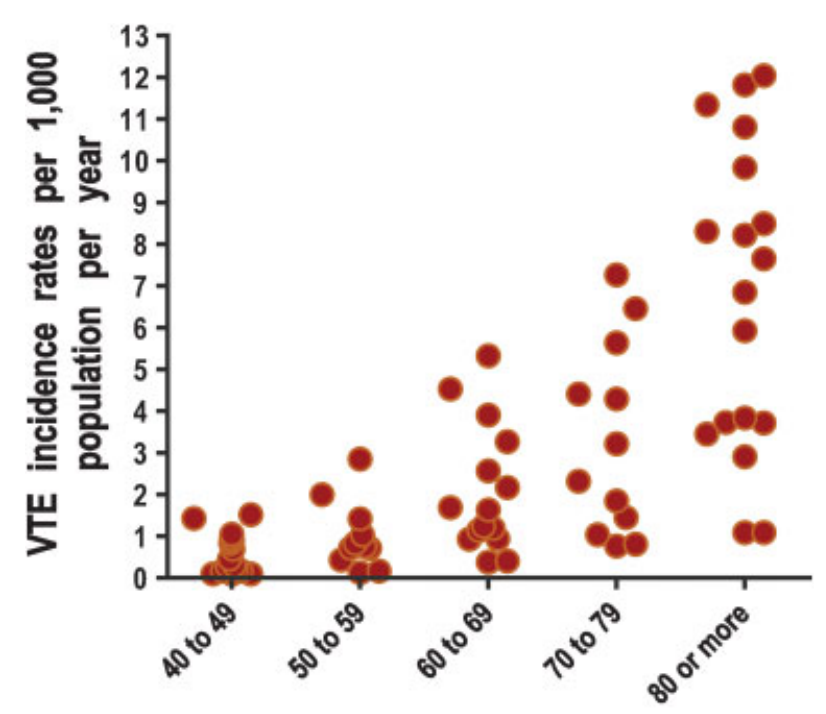

Age range (years)

Fig. 1 Venous thromboembolism (VTE) rates per 1,000 population per year. In general, rates for males were higher than those for females, and rates for certain ethnicities (e.g., Asian, Chinese, Korean and American) were lower than those for whites and African Americans. Finally, there was a strong and consistent association of increasing incidence of VTE with increasing age. Data summarized from the article by Raskob GE, Angchaisuksiri P, Blanco AN, et al; International Society on Thrombosis and Hemostasis (ISTH) Steering Committee for World Thrombosis Day. ${ }^{13}$

Australians versus 1 per 1,000 person-year for Non-indigenous Australians. Of 71, 39 (55\%) of VTE cases in the indigenous group occurred in patients younger than 50 years and almost half of these $(n=18)$ were younger than 29 years. Hospitalization was found to be a major risk factor for VTE in 20 (38\%) of 54 Indigenous Australians of whom 10 (26\%) patients were younger than 50 years. Although the rate of VTE in Indigenous Australians was low, its onset was significantly earlier in life and it was often triggered by prolonged hospitalization. VTE therefore should be added to the list of adverse outcomes of poor health and chronic diseases, which cause disproportional high rates of hospitalization amongst Indigenous Australians. The low number of VTE cases observed in older Indigenous patients in this study possibly reflects on the lower life expectancy and ongoing wide gap in life expectancy between Indigenous and Non-indigenous Australians. In summary, this contribution adds to this issue's lead article by providing new data on VTE incidence in Indigenous Australian people, which is furthermore consistent with lower VTE incidence also observed in some (e.g., Asian) ethnicities, ${ }^{13}$ as well as providing age-related information.

The third contribution in this issue of the journal continues the topic of thrombosis, this time as related to the antiphospholipid (antibody) syndrome (APS). ${ }^{16}$ Patients who are diagnosed with APS are identified to have a high risk of recurrent thrombosis, which can occur despite anticoagulant therapy. APS is an acquired autoimmune syndrome characterized by venous or arterial thrombosis and/or pregnancy morbidity in patients with persistent presence of antiphospholipid antibodies (aPL). The optimal type, intensity, and duration of anticoagulant therapy for the treatment of APS remain controversial issues, particularly for arterial thrombosis and recurrent thrombosis. Patients with persistently positive testing for lupus anticoagulant and elevated levels of anticardiolipin antibodies and anti- $\beta 2$ glycoprotein I antibodies-known as triple positivity-appear to be at increased risk for thrombosis compared with patients who test positive for a single aPL. Recognizing that patients with APS may potentially have different thrombotic risk profiles may assist clinicians in assessing the risks, benefits, and optimal duration of anticoagulation. Although this topic has been previously and extensively covered in this journal, ${ }^{17-20}$ the current review provides an excellent synopsis of the subject matter. Why different aPL define different levels of risks for thrombosis has not been conclusively identified. Various hypotheses have been proposed, including different sensitivities for clinically relevant versus irrelevant antibodies, differences in the level of standardization and harmonization of laboratory assays used to identify the different aPL, as well as the distinct species of aPL defined by each aPL assay. ${ }^{21}$ Whatever the reasons, the author of the current review concludes that future studies that delineate thrombotic risk in APS and evaluate current and novel anticoagulants as well as nonanticoagulant therapies are required. It is also worth noting that this contribution comes from one of the 2014 Eberhard F. Mammen Young Investigator Award winners. ${ }^{12}$

The next contribution by Pennings and Kritharides also continues the topic of thrombosis, also adding the subject of cardiovascular disease, to explore the potential role of CD147, together with its binding partners in platelets, in thrombosis, and arterial disease. ${ }^{22}$ The authors also assess mechanistic aspects of CD147 biology. CD147, also called extracellular matrix metalloproteinase (abbreviated EMMPRIN) is a member of the immunoglobulin superfamily that is expressed on many cell types including hematopoietic, endothelial cells, leukocytes, keratinocytes, platelets, and others. The binding partners of CD147 are numerous and diverse and give some indication to the various roles that CD147 can play; these include homophilic interactions, integrins, cyclophilins (Cyps), glycoprotein VI (GPVI), caveolin-1, and monocarboxylate transporters. Thrombotic and inflammatory pathways play a key role in coronary artery disease (CAD) development. Recent evidence suggests a role for CD147 in both thrombosis and inflammation, as well as involvement in CAD and cancer.

The next contribution by Lippi et $\mathrm{al}^{23}$ continues the thrombosis saga, this time focusing on therapeutic aspects defined by the (newer/novel) direct oral anticoagulants (DOACs), as well as their potential "monitoring," or more appropriately termed laboratory "measurement." The recent development and marketing of these drugs represent a paradigm shift in the management of patients requiring long-term anticoagulation. The advantages of these compounds over traditional therapy with vitamin $\mathrm{K}$ antagonists include a reportedly lower risk of severe hemorrhages and the limited need for laboratory testing. However, there are several scenarios in which testing should be applied. The potential for drug-to-drug interaction is one plausible but currently underrecognized indication for laboratory assessment of 
their anticoagulant effect. In particular, substantial concern has been raised during phase I studies regarding the potential interaction of these drugs with some antibiotics, especially those that interplay with permeability glycoprotein (P-gp) and cytochrome 3A4 (CYP3A4). The authors performed a specific electronic search on clinical trials to confirm that clarithromycin and rifampicin significantly impair the bioavailability of dabigatran, whereas clarithromycin, erythromycin, fluconazole, and ketoconazole alter the metabolism of rivaroxaban in vivo. Because of their more recent development, no published data were found for apixaban and edoxaban, or for potential interactions of DOACs with other and widely used antibiotics. It is noteworthy, however, that an online resource based on Food and Drug Administration (FDA) and social media information, reports several hemorrhagic and thrombotic events in patients simultaneously taking dabigatran and some commonly used antibiotics such as amoxicillin, cephalosporin, and metronidazole. According to these reports, the administration of antibiotics in patients undergoing therapy with DOACs would seem to require accurate evaluation as to whether dose adjustments (personalized or antibiotic class driven) of the anticoagulant drug may be advisable. This might be facilitated by direct laboratory assessments of their anticoagulant effect ex vivo. These DOACs have also been the subject of many previous recent articles in Seminars in Thrombosis $\mathcal{E}$ Hemostasis. ${ }^{24-27}$ of additional interest, there are several guidelines and recommendations for laboratory testing of the DOACs, ${ }^{28}$ which may in time be updated with information such as that explored in this contribution, and which suggests that despite manufacturer-driven dogma that monitoring is not required, that measurements may instead be necessary in many clinical scenarios.

The next contribution begins our journey away from the specific topic of thrombosis and toward the more general field of thrombosis/hemostasis. Danese et al discuss the potential role of nucleic acids (NAs) as unconventional mediators of thrombus formation, intervening in both hemostasis and thrombosis. ${ }^{29}$ NAs constitute the backbone of cellular life, permitting conservation, transmission, and execution of genetic information. In the last few years, new unexpected functions for NAs, projecting them also beyond nuclear and cellular boundaries, have been recognized: circulating cellfree NAs, histones, DNA-histone complexes, microRNAs may have a regulatory role in physiological and pathological processes. Furthermore, in the last decade, the possibility to detect and quantify these in plasma and/or in serum has led to their ancillary use as potential markers in various medical conditions. The use of these as markers within the fields of thrombosis and hemostasis looks especially promising: the potential implications include the possibility to assess patients' risk profiles for thrombotic events and the identification of more directed targets for pharmacologic intervention. The major impediment is that, to date, the methods by which NAs are explored, still largely differ between published studies and standardized procedures remain elusive. The authors propose that future research should focus on the physiological mechanisms underlying the activities of such mediators in specific thrombotic conditions and on the definition of reliable methods for their quantification in biological fluids.

Rabbolini et al then continued the further exploration into hemostasis, this time as related to primary hemostasis, and namely the group of inherited macrothrombocytopenias that remain underrecognized and which are frequently misdiagnosed as immune thrombocytopenia purpura. ${ }^{30}$ Inherited macrothrombocytopenias represent a clinically heterogeneous group of disorders, many of which cause moderateto-severe bleeding tendencies in affected individuals. Diagnostic strategies to date have included a predominant phenotypic approach. The emergence of genetic testing and the implementation of next generation sequencing strategies in the investigation and diagnosis of these disorders have broadened the understanding of their pathogenesis, classification, and presentation. This review describes the increasingly expanding group of recognized inherited macrothrombocytopenias and highlights their pathophysiology and the role of phenotypic and genetic testing in their description and diagnosis.

This issue of Seminars in Thrombosis $\mathcal{E}$ Hemostasis then turns our attention to the topic of other bleeding disorders, specifically for the next three contributions as related to hemophilias. The first of these contributions is from another of our Young Investigator Award winners. ${ }^{12}$ Fernandes et al discuss the issue of prophylaxis as a treatment strategy for severe hemophilia, particularly as prophylaxis started early in life is now well established as the treatment of choice for children with severe hemophilia. ${ }^{31}$ Although, there is no consensus among the hemophilia management community to either stop or maintain prophylactic treatment in adulthood, experts, and centers advise individualized prophylaxis according to clinical bleeding pattern, condition of joints, pharmacokinetic profile, physical activity, type of employment, and patients' personal preferences. The aim of the current report is to describe the impact of an individualized prophylaxis approach on a small cohort of young adults with severe hemophilia, in the setting of a Portuguese Haemophilia Comprehensive Care Centre. The authors explored a tailored prophylaxis approach in this young adult cohort initiated on standard prophylactic regimens in childhood, as based on clinical outcome. Patients were evaluated and prophylaxis was adjusted (dose and/or frequency) to daily life activity and bleeding pattern. After 12 months of follow-up, 9 of 10 patients maintained their new but lowered prophylaxis approach, without increasing bleeding episodes. The authors concluded that with this individualized approach, they observed no negative impact on clinical outcome in most patients, with a proposed improvement in quality of life (given reduced dosing) and a reduction of overall costs such that existing funding could then be utilized to manage an increased number of patients with hemophilia. This contribution may be particularly relevant to economically disadvantaged geographies and promote better utilization of available resources.

The next contribution, by Witkop et $\mathrm{al}^{32}$ continues the theme of hemophilia, but this time from the perspective of 
emerging therapies and the role of the interdisciplinary team in this new era. The authors suggest that the introduction of new hemophilia management therapies, targeting extended half-lives through bioengineering, ushers in an era of potential promise and increasing complexity, more so for those with hemophilia B than hemophilia A. Questions arise for patients, caregivers, and hemophilia treatment center staff about how to assess and incorporate novel therapies and how to determine whether new therapies offer a distinct advantage over established treatment routines. The authors propose that nurses and other interdisciplinary hemophilia treatment center staff are well positioned to assess, educate, and support patients and families in navigating this rapidly changing landscape. To support these challenging efforts, this review offers a perspective on issues affecting therapeutic transitions and provides tools to foster ongoing adherence.

The next contribution, by Tiede et $\mathrm{al}^{33}$ continues the exploration of hemophilia, but this time acquired hemophilia $\mathrm{A}$, which they counsel should be suspected in all patients with a new onset of bleeding and an isolated prolongation of activated partial thromboplastin time (aPTT). About $10 \%$ of acquired hemophilia A patients do not bleed at the time of diagnosis, but they are at risk of future bleeding, in particular, during interventions or surgery. Diagnosis of acquired hemophilia A is confirmed by demonstrating markedly reduced factor VIII activity (FVIII:C) and neutralizing anti-FVIII antibodies, so-called inhibitors. Several limitations and pitfalls exist with the assays used to diagnose acquired hemophilia $\mathrm{A}$, as also extensively explored previously in this journal. ${ }^{34}$ Interference can result from anticoagulants or lupus anticoagulant. ${ }^{35,36}$ The Bethesda assay used to measure inhibitor potency assumes a log-linear relationship between inhibitor concentration and effect on residual FVIII:C activity to allow exact quantification. However, this relationship is not present for the type 2 inhibitors typically seen in acquired hemophilia A. Therefore, this assay only provides a rough estimate of inhibitor potency. These limitations can explain, in part, why laboratory data, such as inhibitor potency, failed to predict bleeding or response to treatment in acquired hemophilia A. This article reviews the diagnostic approach to acquired hemophilia A, discusses assay-specific limitations, including limitations of mixing tests, ${ }^{37}$ and addresses some of the challenges for future research.

The final contribution to this issue of Seminars in Thrombosis E Hemostasis is by Lippi et al, ${ }^{38}$ and discusses the important topic of "defensive medicine," otherwise largely unexplored within the field of thrombosis and hemostasis. "Defensive medicine," as a term, conventionally defines the medical practice of ordering medically questionable diagnostic testing, procedures, or visits, or to avoid high-risk patients or procedures, potentially as aimed to reduce exposure to malpractice liability, to avoid patient criticism regarding "medical inaction," or to avoid "missing" some otherwise potential identifiable defect(s). Although the precise impact of defensive medicine in the field of laboratory testing is difficult to estimate from the current literature, the authors report that overuse or inappropriate use of labora- tory resources ranges from 23 to $67 \%$, and a large part of this can be attributed to medical liability concerns, with apparently little clinical awareness of the adverse consequences that may be associated with this practice. Essentially, performing inappropriate testing remarkably increases the risk of obtaining false-positive results due to statistical, preanalytical, and analytical reasons, thus triggering further and potentially even more invasive follow-up testing, inappropriate patient management, along with incremental increases of expenditure due to misuse of health care resources. Although one pragmatic example is the overuse of prostate-specific antigen testing, as routine coagulation testing is commonly performed for the screening of patients with bleeding or thrombotic disorders, either a false-negative or a false-positive result in these basic procedures may also significantly impact on clinical outcomes and health care resources. The primary aim of this article is to describe the leading causes of physiological, pathological, therapeutic, and spurious variations of the prothrombin time, aPTT, and D-dimer, as well as the potential clinical consequences emerging from the generation of false-negative and falsepositive results with these tests. The authors plan to further explore these problems in subsequent issues of this journal and as relating to more specialized "diagnostic" assays of hemostasis.

I thank all the authors to this issue of Seminars in Thrombosis \& Haemostasis for their original and comprehensive contributions. However, I especially thank the Eberhard F. Mammen Young Investigator Award winners for their submissions, as well as again highlighting the lead article in this issue. Ultimately, however, I hope that the readership of this journal finds the entire issue of considerable interest. This will of course be determined in time, as measured and established for previous issues of "hot topic" compilations. ${ }^{1-11}$

\section{References}

1 Favaloro EJ. Hot topics I: a potpourri of current issues and controversies in thrombosis and hemostasis. Semin Thromb Hemost 2007;33(8):723-726

2 Favaloro EJ. Hot topics II: an editorial collection of current issues and controversies in thrombosis and hemostasis. Semin Thromb Hemost 2008;34(1):3-6

3 Favaloro EJ. Hot topics III. Preface. Semin Thromb Hemost 2012; $38(1): 1-4$

4 Favaloro EJ. Hot Topics IV. Semin Thromb Hemost 2013;39(1):1-4

5 Favaloro EJ. Hot topics V. Preface. Semin Thromb Hemost 2014; 40(1):5-10

6 Favaloro EJ. Editorial. Winners of the inaugural Eberhard F. Mammen Award for most popular article. Semin Thromb Hemost 2009; 35:587-590

7 Favaloro EJ. Winners of the 2010 Eberhard F. Mammen award for most popular article during 2008-2009. Semin Thromb Hemost 2010;36(7):685-692

8 Favaloro EJ. 2011 Eberhard F. Mammen award announcements. Semin Thromb Hemost 2011;37(5):431-439

9 Favaloro EJ. 2012 Eberhard F. Mammen award announcements. Semin Thromb Hemost 2012;38(5):425-432

10 Favaloro EJ. 2013 Eberhard F. Mammen award announcements. Semin Thromb Hemost 2013;39(6):567-574 
11 Favaloro EJ. 2014 Eberhard F. Mammen award announcements: Part I - most popular articles. Semin Thromb Hemost 2014;40(4): 407-412

12 Favaloro EJ. 2014 Eberhard F. Mammen Award announcements: part II-Young Investigator Awards. 2014;40(7):718-723

13 Raskob GE, Angchaisuksiri P, Blanco AN, et al; ISTH Steering Committee for World Thrombosis Day. Thrombosis: a major contributor to global disease burden. Semin Thromb Hemost 2014;40(7):724-735

14 Kwaan HC, McMahon BJ, Hylek EM. Age-related changes in thrombosis and hemostasis. Semin Thromb Hemost 2014;40(6): 619-620

15 Szabo F, Marshall C, Huynh DK. Venous thromboembolism in tropical Australia and in Indigenous Australians. Semin Thromb Hemost 2014;40(7):736-740

16 Lim W. Thrombotic risk in the antiphospholipid syndrome. Semin Thromb Hemost 2014;40(7):741-746

17 Favaloro EJ, Wong RC. The antiphospholipid syndrome: diagnosis, pathogenesis, laboratory testing, and management. Semin Thromb Hemost 2012;38(4):299-304

18 Pengo V, Banzato A, Bison E, Bracco A, Denas G, Ruffatti A. What have we learned about antiphospholipid syndrome from patients and antiphospholipid carrier cohorts? Semin Thromb Hemost 2012;38(4):322-327

19 Les I, Ruiz-Irastorza G, Khamashta MA. Intensity and duration of anticoagulation therapy in antiphospholipid syndrome. Semin Thromb Hemost 2012;38(4):339-347

20 Galli M. Interpretation and recommended testing for antiphospholipid antibodies. Semin Thromb Hemost 2012;38(4):348-352

21 Favaloro EJ, Wong RCW. Antiphospholipid antibody testing for the antiphospholipid syndrome: a comprehensive practical review including a synopsis of challenges and recent guidelines. Pathology 2014;46(6):481-495

22 Pennings GJ, Kritharides L. CD147 in cardiovascular disease and thrombosis. Semin Thromb Hemost 2014;40(7):747-755

23 Lippi G, Favaloro EJ, Mattiuzzi C. Combined administration of antibiotics and direct oral anticoagulants: a renewed indication for laboratory monitoring? Semin Thromb Hemost 2014;40(7): 756-765

24 Salmela B, Joutsi-Korhonen L, Armstrong E, Lassila R. Active online assessment of patients using new oral anticoagulants: bleeding risk, compliance, and coagulation analysis. Semin Thromb Hemost 2012;38(1):23-30

25 Rojas-Hernandez CM, Garcia DA. The novel oral anticoagulants. Semin Thromb Hemost 2013;39(2):117-126
26 Di Minno A, Spadarella G, Prisco D, Franchini M, Lupoli R, Di Minno MN. Clinical judgment when using coagulation tests during direct oral anticoagulant treatment: a concise review. Semin Thromb Hemost 2013;39(7):840-846

27 Harenberg J, Du S, Krämer S, et al. Novel methods for assessing oral direct factor $\mathrm{Xa}$ and thrombin inhibitors: use of point-of-care testing and urine samples. Semin Thromb Hemost 2013;39(1): 66-71

28 Lippi G, Favaloro EJ. Recent guidelines and recommendations for laboratory assessment of the direct oral anticoagulants (DOACs): Is there consensus? Clin Chem Lab Med 2014; doi: 10.1515/cclm2014-0767

29 Danese E, Montagnana M, Fava C, Guidi GC. Circulating nucleic acids and hemostasis: biological basis behind their relationship and technical issues in assessment. Semin Thromb Hemost 2014; 40(7):766-773

30 Rabbolini DJ, Morel-Kopp MC, Stevenson W, Ward CM. Inherited macrothrombocytopenias. Semin Thromb Hemost 2014;40(7): 774-784

31 Fernandes S, Carvalho M, Lopes M, Araújo F. Impact of an individualised prophylaxis approach on young adults with severe haemophilia. Semin Thromb Hemost 2014;40(7):785-789

32 Witkop M, Cutter S, Deutsche J, Santaella M, Chapman R, Lafranco J, Lambing A. Emerging therapies for hemophilia: a new era of care and the role of the interdisciplinary team. Semin Thromb Hemost 2014;40(7):790-802

33 Tiede A, Werwitzke S, Scharf RE. Laboratory diagnosis of acquired hemophilia a: limitations, consequences, and challenges. Semin Thromb Hemost 2014;40(7):803-811

34 Favaloro EJ, Meijer P, Jennings I, et al. Problems and solutions in laboratory testing for hemophilia. Semin Thromb Hemost 2013; 39(7):816-833

35 Kershaw G, Suresh S, Orellana D, Nguy YM. Laboratory identification of lupus anticoagulants. Semin Thromb Hemost 2012;38(4): 375-384

36 Kershaw G, Jayakodi D, Dunkley S. Laboratory identification of factor inhibitors: the perspective of a large tertiary hemophilia center. Semin Thromb Hemost 2009;35(8):760-768

37 Kershaw G, Orellana D. Mixing tests: diagnostic aides in the investigation of prolonged prothrombin times and activated partial thromboplastin times. Semin Thromb Hemost 2013;39(3):283-290

38 Lippi G, Favaloro EJ, Franchini M. Dangers in the practice of defensive medicine in hemostasis testing for investigation of bleeding or thrombosis: Part I-routine coagulation testing. Semin Thromb Hemost 2014;40(7):812-824 